\title{
Harnack Inequality and Applications for Stochastic Retarded Differential Equations Driven by Fractional Brownian Motion
}

\author{
LIU Min, XU Liping*, LI Zhi and CHEN Zhong \\ School of Information and Mathematics, Yangtze University, Jingzhou 434023, China.
}

Received 3 November 2016; Accepted 20 December 2016

\begin{abstract}
In this paper, by using a semimartingale approximation of a fractional stochastic integration, the global Harnack inequalities for stochastic retarded differential equations driven by fractional Brownian motion with Hurst parameter $0<H<1$ are established. As applications, strong Feller property, log-Harnack inequality and entropycost inequality are given.
\end{abstract}

AMS Subject Classifications: 60G15, 60H05, 60H15

Chinese Library Classifications: $\mathrm{O} 211.63$

Key Words: Fractional Brownian motion; Harnack inequality; strong Feller property.

\section{Introduction}

Under a curvature condition, Wang [1] established the following type dimension-free Harnack inequality for diffusion semigroups on a Riemannian manifold $M$ :

$$
\left(P_{t} f\right)^{\alpha}(y) \leq\left(P_{t} f^{\alpha}\right)(x) e^{c(t) \rho(x, y)^{2}}, \quad f \geq 0, t>0, \alpha>1, x, y \in M,
$$

where $c(t)>0$ is explicitly determined by $\alpha$ and the curvature lower bound. This type of inequality has been studied extensively, see, for example, Aida and Kawabi [2] and Aida and Zhang [3] for infinite dimensional diffusion processes; Wang [4] for stochastic generalized porous media equations; Röckner and Wang [5] for generalizes Mehler semigroup; Abdelhadi et.al. [6] and Wang and Yuan [7] for stochastic functional equation; Liu [8] for stochastic evolution equations with monotone drifts; Ouyang [9] for Ornstein-Uhnelbeck processes and multivalued stochastic evolution equations etc.

*Corresponding author. Email addresses: xlp211@126.com (L. Xu), fqbu@yangtzeu.edu.cn (M. Liu), lizhi_csu@126.com (Z. Li), czhong@yangtzeu.edu.cn (Z. Chen) 
The Harnack inequality has various applications, see for instance, [5,10-12] for strong Feller property and contractivity properties; [2,3] for short times behaviors of infinite dimensional diffusions; [13-15] for heat kernel estimates and entropy-cost inequalities. $[1,5,16]$ established Harnack inequalities using the method of derivative formula. In order to establish the Harnack inequality for diffusions with curvature unbounded below, the approach of coupling and Girsanov transformations are developed in [17]. This method works also for infinite dimensional SPDE provided the noise is additive and non-degenrate, see e.g. $[4,9,18,19]$ for Harnack inequalities for several different classes of SPDE.

On the other hand, one solution for many SDEs is a semimartingale as well a Markov process. However, many objects in real world are not always such processes since they have long-range aftereffects. Since the work of Mandelbrot and Van Ness [20], there has been an increased interest in stochastic models based on the fractional Brownian motion than Brownian motion. A fractional Brownian motion (fBm) of Hurst parameter $H \in(0,1)$ is a centered Gaussian process $B^{H}=\left\{B^{H}(t), t \geq 0\right\}$ with the covariance function

$$
R_{H}(t, s)=\mathbb{E}\left(B_{t}^{H} B_{s}^{H}\right)=\frac{1}{2}\left(t^{2 H}+s^{2 H}-|t-s|^{2 H}\right) .
$$

When $H=1 / 2$ the $\mathrm{fBm}$ becomes the standard Brownian motion, and the $\mathrm{fBm} B^{H}$ neither is a semimartingale nor a Markov process if $H \neq 1 / 2$. However, the $\mathrm{fBm} B^{H}, H>1 / 2$ is a long-memory process and presents an aggregation behavior. The long-memory property make $\mathrm{fBm}$ as a potential candidate to model noise in mathematical finance (see [21]); in biology (see [22]); in communication networks (see, for instance [23]); the analysis of global temperature anomaly [24] and electricity markets [25] etc.

Very recently, using derivative formula, the local Harnack inequalities in the sense that $|x-y|$ is bounded by a constant for the following stochastic differential equations

$$
\mathrm{d} X(t)=b(X(t)) \mathrm{d} t+\mathrm{d} B^{H}(t), \quad X(0)=x,
$$

driven by fractional Brownian motion with Hurst parameter $1 / 2<H<1$ were established by Fan in [26]. Subsequently, using the approach of coupling and Girsanov transformations to fractional Brownian motion with Hurst parameter $1 / 2<H<1$, the global Harnack inequalities for the following stochastic differential equations

$$
\mathrm{d} X(t)=b(t, X(t)) \mathrm{d} t+\sigma(t) \mathrm{d} B^{H}(t), \quad X(0)=x,
$$

driven by fractional Brownian motion with Hurst parameter $1 / 2<H<1$ were established by Fan in [27]. Furthermore, using Malliavin calculus, Fan [28] established Bismut derivative formulae for the following stochastic differential equations

$$
\mathrm{d} X(t)=b(X(t)) \mathrm{d} t+\sigma(t) \mathrm{d} B^{H}(t), \quad X(0)=x,
$$

and functional stochastic differential equations

$$
\mathrm{d} X(t)=b\left(X_{t}\right) \mathrm{d} t+\sigma(t) \mathrm{d} B^{H}(t), \quad X_{0}=\xi ，
$$


driven by fractional Brownian motions with Hurst parameter $1 / 2<H<1$. As applications, the global Harnack type inequalities are presented.

However, in [28] the condition that $b$ is Fréchet differentiable such that $\nabla b$ is bounded and Lipschitz continuous seems to be relatively strong. On the other hand, as far as I know, in the case that $1 / 2<H<1$, using the approach of coupling for the segment process and Girsanov transformations, in virtue of irregularity of the operator $K_{H}^{-1}$, it is very difficult to obtain the Harnack inequality. In [20], Mandelbrot et al. have given a representation of $B_{t}^{H}$ of the form:

$$
B_{t}^{H}=\frac{1}{\Gamma(1+\alpha)}\left(U(t)+\int_{0}^{t}(t-s)^{\alpha} \mathrm{d} W_{s}\right),
$$

where $\alpha=H-1 / 2, U(t)$ is a stochastic process of absolutely continuous trajectories, and $W_{t}^{H}:=\int_{0}^{t}(t-s)^{\alpha} \mathrm{d} W_{s}$ is called a fBm of the Liouville form $(\mathrm{LfBm})$. Because a LfBm shares many properties of a $\mathrm{fBm}$ (except that it has non-stationary increments) and for simplicity we use $W_{t}^{H}$ standing for $B_{t}^{H}$ throughout this paper. In this paper, motivating mainly by [29], using a semimartingale approximation of a fractional stochastic integration, we desire to establish the global Harnack inequality for the segment process to the following stochastic functional differential equations

$$
\left\{\begin{array}{l}
\mathrm{d} X(t)=b(X(t)) \mathrm{d} t+F\left(X_{t}\right) \mathrm{d} t+\sigma(t) \mathrm{d} W_{t}^{H}, \\
X_{0}=\xi,
\end{array}\right.
$$

driven by fractional Brownian motion with Hurst parameter $0<H<1$. As applications, strong Feller property, log- Harnack inequality and entropy-cost inequality are derived.

The paper is organized as follows. In Section 2, we give some preliminaries on fractional Brownian motion. In Section 3, we establish the Harnack inequality, and present some applications.

\section{Preliminaries}

In the last few decades, many differential ways have been introduced to constructed the fractional stochastic calculus (see, for instance, [30]). The main difficulties in studying fractional stochastic systems are that we cannot apply stochastic calculus developed by Itô since $\mathrm{fBm}$ is neither a Markov process nor a semimartingale, except for $H=1 / 2$. Recently, an approximate approach has been developed to avoid those difficulties (see, $[29,31]$ and the references therein). Let us recall some fundamental results about this approach.

Let $\left(\Omega, \mathcal{F},\left\{\mathcal{F}_{t}\right\}_{t \geq 0}, \mathbb{P}\right)$ be a filtered complete probability space satisfying the usual condition, which means that the filtration is a right continuous increasing family and $\mathcal{F}_{0}$ contains all $P$-null sets. For every $\varepsilon>0$ we define

$$
W_{t}^{H, \varepsilon}=\int_{0}^{t}(t-s+\varepsilon)^{\alpha} \mathrm{d} W_{s} .
$$


In [31], author proved that $W_{t}^{H, \varepsilon}$ is a semimartingale with the following decomposition

$$
W_{t}^{H, \varepsilon}=\varepsilon^{\alpha} W_{t}+\int_{0}^{t} \varphi^{\varepsilon}(s) \mathrm{d} s,
$$

where $\varphi^{\varepsilon}(s)=\int_{0}^{s} \alpha(s+\varepsilon-u)^{\alpha-1} \mathrm{~d} W_{u}$. Moreover, $W_{t}^{H, \varepsilon}$ converges to $W_{t}^{H}$ in $L^{p}(\Omega), p>1$ uniformly in $t \in[0, T]$ as $\varepsilon \rightarrow 0$ :

$$
\mathbb{E}\left|W_{t}^{H, \varepsilon}-W_{t}^{H}\right|^{p} \leq c_{p, T} \varepsilon^{p H} .
$$

For $f$ is a deterministic function in $L^{2}[0, T]$, from the decomposition (2.1) we have

$$
\begin{aligned}
\int_{0}^{t} f(s) \mathrm{d} W_{s}^{H, \varepsilon} & =\int_{0}^{t} \varepsilon^{\alpha} f(s) \mathrm{d} W_{s}+\int_{0}^{t} \int_{0}^{s} \alpha f(s)(s+\varepsilon-u)^{\alpha-1} \mathrm{~d} W_{u} \mathrm{~d} s \\
& =\int_{0}^{t} \varepsilon^{\alpha} f(s) \mathrm{d} W_{s}+\int_{0}^{t} \int_{s}^{t} \alpha f(u)(u+\varepsilon-s)^{\alpha-1} \mathrm{~d} u \mathrm{~d} W_{s} .
\end{aligned}
$$

As $\varepsilon \rightarrow 0$, each term in the right-hand side of (2.2) converges in $L^{2}(\Omega)$ to the same term where $\varepsilon=0$. Then, it is 'natural' to define.

Definition 2.1. For $f$ is a deterministic function in $L^{2}[0, T]$. The stochastic integral of $f$ with respect to $L f B m$ is defined by

$$
\int_{0}^{t} f(s) \mathrm{d} W_{s}^{H}:=\lim _{\varepsilon \rightarrow 0} \int_{0}^{t} f(s) \mathrm{d} W_{s}^{H, \varepsilon}=\alpha \int_{0}^{t} \int_{s}^{t} \alpha f(u)(u-s)^{\alpha-1} \mathrm{~d} u \mathrm{~d} W_{s} .
$$

Let $r>0$ be fixed, and let $\mathcal{L}=C([-r, 0] ; \mathbb{R})$ be equipped with the uniform norm $\|\xi\|_{\infty}=$ $\sup _{-r \leq t \leq 0}|\xi(t)|$. We consider the following functional stochastic differential equation driven by fractional Brownian motion on $\mathbb{R}$,

$$
\left\{\begin{array}{l}
\mathrm{d} X(t)=b(X(t)) \mathrm{d} t+F\left(X_{t}\right) \mathrm{d} t+\sigma(t) \mathrm{d} W_{t}^{H}, \\
X_{0}=\xi,
\end{array}\right.
$$

where $\xi \in \mathcal{L}$, for each $t \geq 0, X_{t} \in \mathcal{L}$ is fixed as $X_{t}(u)=X(t+u), u \in[-r, 0]$.

The aim of the paper is to consider the Harnack inequality for the equation (2.4). We define

$$
P_{t} f(\xi):=\mathbb{E} f\left(X_{t}^{\xi}\right), \quad t \in[0, T], f \in \mathscr{B}_{b}(\mathcal{L}),
$$

where $X_{t}^{\xi}$ is the solution to the equation $(2.2)$ and $\mathscr{B}_{b}(\mathcal{L})$ denotes the set of all bounded measurable functions on $\mathcal{L}$.

\section{Harnack inequality}

Let us start with the following hypotheses: 
(H1) $|b(x)-b(y)| \leq K_{1}|x-y|, \forall x, y \in \mathbb{R}$, where $K_{1}>0$ is a constant;

(H2) $F$ is globally Lipschitz on $\mathcal{L}$, i.e. for some $K_{2}>0$,

$$
|F(x)-F(y)| \leq K_{2}\|x-y\|_{\infty}, \quad \forall x, y \in \mathcal{L}, t \in[0, T] .
$$

(H3) $\sigma(\cdot)$ is reversible and the inverse is bounded, i.e. for any $t \in \mathbb{R}$, there exists a constant $M>0$ such that $\left|\sigma^{-1}\right| \leq M$.

Remark 3.1. For $\sigma$ is a deterministic function in $L^{2}[0, T]$. Using the same arguments as in [32], we can easily prove that there exists a unique strong solution to equation (2.4) under the assumption (H1) and (H2).

Now, we aim to establish Harnack inequality for $P_{T}$.

Theorem 3.1. Let $T>r, 0<H<1$. If (H1)-(H3) hold, then the operator $P_{T}$ satisfies that for any $p>1, \xi, \eta \in \mathcal{L}$ and nonnegative $f \in B_{b}(\mathcal{L})$,

$$
\left(P_{T} f(\eta)\right)^{p} \leq P_{T} f^{p}(\xi) \exp \left[\frac{p}{p-1} \rho(T, r, H, \xi, \eta)\right],
$$

where

$$
\begin{aligned}
& \rho(T, r, H, \xi, \eta)=\inf _{r<s \leq T} \rho(T, r, s, H, \xi, \eta), \\
& \rho(T, r, s, H, \xi, \eta)=M \cdot\left\{K_{2}\|\xi-\eta\|_{\infty}+K_{2} e^{K_{1} s}|\xi(0)-\eta(0)|+\frac{K_{1}|\xi(0)-\eta(0)|}{\left(1-e^{-K_{1}(s-r)}\right)}\right\}^{2} \frac{T^{2-2 H}}{2-2 H} .
\end{aligned}
$$

Proof. The proof will be divided into three steps.

Step 1. As in [6] we shall employ a coupling argument. Let

$$
G(x)= \begin{cases}\frac{x}{|x|}|x|^{\varepsilon}, & \text { if } x \neq 0 \\ 0, & \text { if } x=0\end{cases}
$$

Let process $(Y(t))_{t \geq 0}$ solve the equation

$$
\left\{\begin{array}{l}
\mathrm{d} Y(t)=b(Y(t)) \mathrm{d} t+F\left(X_{t}\right) \mathrm{d} t+\gamma \cdot G(X(t)-Y(t)) \mathrm{d} t+\sigma(t) \mathrm{d} B^{H}(t) \\
Y(0)=\eta(0)
\end{array}\right.
$$

and which we extend by $Y(t)=\eta(t)$ for $t \in[-r, 0)$. Note that

$$
\mathrm{d}(X(t)-Y(t))=(b(X(t))-b(Y(t))) \mathrm{d} t-\gamma \cdot G(X(t)-Y(t)) \mathrm{d} t .
$$

Thus applying the Tanaka formula to $|X(t)-Y(t)|$, we have

$$
\begin{aligned}
\mathrm{d}|X(t)-Y(t)| & =\operatorname{sgn}(X(t)-Y(t)) \mathrm{d}(X(t)-Y(t)) \\
& =\operatorname{sgn}(X(t)-Y(t))(b(X(t))-b(Y(t))) \mathrm{d} t-\gamma \cdot|X(t)-Y(t)|^{\varepsilon} \mathrm{d} t .
\end{aligned}
$$


In view of (H1), for all $t \geq 0$ we get

$$
\mathrm{d}|X(t)-Y(t)| \leq K_{1}|X(t)-Y(t)| \mathrm{d} t-\gamma \cdot|X(t)-Y(t)|^{\varepsilon} \mathrm{d} t .
$$

This implies that

$$
\mathrm{d}\left(e^{-K_{1} t}|X(t)-Y(t)|\right) \leq-\gamma e^{-K_{1} t} \cdot|X(t)-Y(t)|^{\varepsilon} \mathrm{d} t .
$$

Then, by virtue of the above inequality and the chain rule, we have that

$$
\begin{aligned}
\mathrm{d}\left(e^{-K_{1} t}|X(t)-Y(t)|\right)^{1-\varepsilon} & =(1-\varepsilon)\left(e^{-K_{1} t}|X(t)-Y(t)|\right)^{-\varepsilon} \mathrm{d}\left(e^{-K_{1} t}|X(t)-Y(t)|\right) \\
& \leq-\gamma(1-\varepsilon) e^{-K_{1} t(1-\varepsilon)} \mathrm{d} t \\
\left(e^{-K_{1} t}|X(t)-Y(t)|\right)^{1-\varepsilon} \leq & \left(|\zeta(0)-\eta(0)|^{1-\varepsilon}-\frac{\gamma}{K_{1}}\left(1-e^{-K_{1} t(1-\varepsilon)}\right)\right)_{+}
\end{aligned}
$$

Thus,

$$
|X(t)-Y(t)| \leq e^{K_{1} t}\left(|\xi(0)-\eta(0)|^{1-\varepsilon}-\frac{\gamma}{K_{1}}\left(1-e^{-K_{1} t(1-\varepsilon)}\right)\right)_{+}^{1 /(1-\varepsilon)} .
$$

Hence, for $s \in(r, T]$, choosing

$$
\gamma=\gamma_{s}=\frac{K_{1}|\xi(0)-\eta(0)|^{1-\varepsilon}}{\left(1-e^{-K_{1}(1-\varepsilon)(s-r)}\right)}>0 .
$$

This implies $X(t)=Y(t)$ for all $t \geq s-r$ and $X_{t}=Y_{t}$ in $\mathcal{C}$ for all $t \geq s$.

Step 2. Let

$$
u(t)=F\left(X_{t}\right)-F\left(Y_{t}\right)+\gamma \cdot G(X(t)-Y(t)), \quad \widetilde{W}_{t}^{H}=\int_{0}^{t} \sigma^{-1}(v) u(v) \mathrm{d} v+W_{t}^{H} .
$$

Note that

$$
|u(t)| \leq K_{2}\left\|X_{t}-Y_{t}\right\|_{\infty}+\gamma|X(t)-Y(t)|^{\varepsilon}
$$

Thus, we have

$$
|u(t)| \leq K_{2}\|\xi-\eta\|_{\infty}+K_{2} \sup _{0 \leq v \leq t}|X(v)-Y(v)|+\gamma|X(t)-Y(t)|^{\varepsilon} .
$$

Finally, letting $\varepsilon \rightarrow 0$, we obtain by (3.3)

$$
|u(t)| \leq K_{2}\|\xi-\eta\|_{\infty}+K_{2} e^{K_{1} s}|\xi(0)-\eta(0)|+\frac{K_{1}|\xi(0)-\eta(0)|}{\left(1-e^{-K_{1}(s-r)}\right)} .
$$


According to integral representation of fractional Brownian motion we deduce for any $t \in[0, T]$

$$
\begin{aligned}
\widetilde{W}_{t}^{H} & =\int_{0}^{t} \sigma^{-1}(v) u(v) \mathrm{d} v+W_{t}^{H} \\
& =\int_{0}^{t} \sigma^{-1}(v) u(v) \mathrm{d} v+\int_{0}^{t}(t-v)^{\alpha} \mathrm{d} W(v) \\
& =\int_{0}^{t}(t-v)^{\alpha}\left(\sigma^{-1}(v) u(v)(t-v)^{-\alpha} \mathrm{d} v+\mathrm{d} W(v)\right) \\
& =: \int_{0}^{t}(t-v)^{\alpha} \mathrm{d} \widetilde{W}(v) .
\end{aligned}
$$

Now, let

$$
R_{\varepsilon}(t)=\exp \left[-\int_{0}^{t} \sigma^{-1}(v) u(v)(t-v)^{-\alpha} \mathrm{d} W(v)-\frac{1}{2} \int_{0}^{t}\left(\sigma^{-1}(v) u(v)(t-v)^{-\alpha}\right)^{2} \mathrm{~d} v\right] .
$$

We want to show that $\left(\widetilde{W}_{t}^{H}\right)_{0 \leq t \leq T}$ is an $\mathcal{F}_{t}^{W^{H}}$-fractional Brownian motion with Hurst parameter $H \in(0,1)$ under the new probability $\mathbb{Q}(d \omega)=R_{\varepsilon}(t) \mathbb{P}(d \omega)$. Due to [33], it suffices to show that $\mathbb{E}^{\mathbb{P}} R_{\varepsilon}(T)=1$. Notice that

$$
\begin{aligned}
& \int_{0}^{T}\left(\sigma^{-1}(v) u(v)(T-v)^{-\alpha}\right)^{2} \mathrm{~d} v \\
\leq & \left\|\sigma^{-1}\right\|_{\infty} \cdot\left\{K_{2}\|\xi-\eta\|_{\infty}+K_{2} e^{K_{1} s}|\xi(0)-\eta(0)|+\frac{K_{1}|\xi(0)-\eta(0)|}{\left(1-e^{-K_{1}(s-r)}\right)}\right\}^{2} \cdot \frac{T^{2-2 H}}{H-2 H} \\
= & : 2\left\|\sigma^{-1}\right\|_{\infty} \rho(T, r, s, H, \varepsilon, \eta) .
\end{aligned}
$$

As a consequence, we get

$$
\mathbb{E} \exp \left[\frac{1}{2} \int_{0}^{T}\left(\sigma^{-1}(v) u(v)(T-v)^{-\alpha}\right)^{2} \mathrm{~d} v\right] \leq \exp [\rho(T, r, s, H, \xi, \eta)] .
$$

Using the well-known Novikov criterion, one can have $\mathbb{E}^{\mathbb{P}} R_{\varepsilon}(T)=1$.

Step 3. From step 2, we can rewrite (3.2) in the following form

$$
\left\{\begin{array}{l}
\mathrm{d} Y(t)=b(Y(t)) \mathrm{d} t+F\left(Y_{t}\right) \mathrm{d} t+\sigma(t) \mathrm{d} \widetilde{W}_{t}^{H}, \\
Y_{0}=\eta
\end{array}\right.
$$

where $\left(\widetilde{W}_{t}^{H}\right)_{0 \leq t \leq T}$ is an $\mathcal{F}_{t}^{W^{H}}$-fractional Brownian motion with Hurst parameter $H$ under the new probability $R_{\varepsilon}(T) P$. By the uniqueness of the solution and $X_{T}=Y_{T}$, a.s., we have

$$
P_{T} f(\eta)=\mathbb{E}^{\mathbb{Q}} f\left(Y_{T}^{\eta}\right)=\mathbb{E}^{\mathbb{Q}} f\left(X_{T}^{\xi}\right)=\mathbb{E}^{\mathbb{P}} R_{\varepsilon}(T) f\left(X_{T}^{\xi}\right) .
$$


Applying the Hölder's inequality to (3.8), we obtain

$$
\left(P_{T} f(\eta)\right)^{p} \leq P_{T} f^{p}(\xi) \cdot\left(\mathbb{E} R_{\varepsilon}(T)^{\frac{p}{p-1}}\right)^{p-1} .
$$

Now we will estimate moments of $R_{\varepsilon}(T)$.

Denote $\alpha=p /(p-1)$ and $M_{T}=-\int_{0}^{T} \sigma^{-1}(v) u(v)(t-v)^{-\alpha} \mathrm{d} W_{v}$. Since $\left(R_{\varepsilon}(t)\right)_{0 \leq t \leq T}$ is a $\mathbb{P}$ martingale, by (3.7) we have

$$
\begin{aligned}
\mathbb{E} R_{\varepsilon}(T)^{\alpha} & =\operatorname{Eexp}\left[\alpha M_{T}-\frac{1}{2} \alpha\langle M\rangle_{T}\right] \\
& =\operatorname{Eexp}\left[\alpha M_{T}-\frac{1}{2} \alpha^{2}\langle M\rangle_{T}+\frac{1}{2} \alpha(\alpha-1)\langle M\rangle_{T}\right] \\
& \leq \exp [\alpha(\alpha-1) \rho(T, r, H, \xi, \eta)] .
\end{aligned}
$$

Substituting (3.10) into (3.9), we get the desired result. The proof is complete now.

Remark 3.2. In [26], Fan established Harnack inequality for equation (2.4) of high dimensions and multiplicative noise in one-dimension without delay by using the method of derivative formula. However, the Harnack inequality established in [26] is local in the sense that $|x-y|$ is bounded by a constant. The Harnack inequality established in our Theorem 3.1 is global. Furthermore, in our Theorem 3.1, the condition that

$$
\nabla b(x)-\nabla b(y) \leq K|x-y|, \quad \forall x, y \in \mathbb{R},
$$

is not required. Incidentally, when the condition $\nabla b(x)-\nabla b(y) \leq K|x-y|$ is dropped even in high dimensions, Fan [27] also established global Harnack inequality for equation (2.4) without delay by using the approach of coupling and Girsanov transformations to fractional Brownian motion with Hurst parameter $1 / 2<H<1$.

For the operator $P_{T}, T \geq 0$, defined in (2.5), it is called strongly Feller if $P_{T}$ maps $B_{b}(\mathcal{L})$ into $C_{b}(\mathcal{L})$ for each $t>0$. Here $C_{b}(\mathcal{L})$ is the space of all bounded continuous functions on $\mathcal{L}$. In general, the operator $P_{T}, T \geq 0$, given in (2.5) might not have strongly Feller property for all $T>0$. However, as a direct application of the Harnack type inequalities derived above, by Proposition 3.1 of [4] we get the strong Feller property on $P_{T}$.

Corollary 3.1. Under the same conditions as in Theorem 3.1, the operator $P_{T}, T \geq 0$, given in (2.5) is eventual strongly Feller in the sense that $P_{T} f(\cdot)$ is a bounded continuous function on $\mathcal{L}$ for each $f \in B_{b}(\mathcal{L})$ and $T>r$. Moreover, we have the following estimate,

$$
\left|P_{T} f(\xi)-P_{T} f(\eta)\right| \leq\|f\|_{\infty}[2 \rho(T, r, H, \xi, \eta)]^{1 / 2} \exp [\rho(T, r, H, \xi, \eta)]
$$

for every $T>r, \xi, \eta \in \mathcal{L}$ and $f \in B_{b}(\mathcal{L})$.

As an immediate application of Theorem 3.1, by Corollary 1.2 of [34], we may also establish the following result on log-Harnack inequality. 
Corollary 3.2. Under the same conditions as in Theorem 3.2, we have the following log-Harnack inequality,

$$
P_{T}(\log f)(\eta) \leq \log P_{T} f(\xi)+\rho(T, r, H, \xi, \eta)
$$

for any $\xi, \eta \in \mathcal{L}, T>r$ and $f \in B_{b}(\mathcal{L})$ satisfying $\inf _{\xi \in \mathcal{L}}|f(\xi)| \geq 1$.

To state further application of Theorem 3.2, let us introduce another assumption and some notations.

(H4): let $\mu$ be a probability measure on $\mathcal{L}$ such that for some $\widetilde{K}>0$,

$$
\mu\left(P_{T} f\right) \leq \widetilde{K} \mu(f), \quad \forall f \in \mathscr{B}_{b}^{+}(\mathcal{L}) .
$$

Note that if $\mu$ is $P_{T}$-invariant, then (H4) holds.

Remark 3.3. The measure $\mu$ satisfying (H4) always exist. For instance (see [35]),

$$
\mu=\sum_{n=1}^{\infty} \frac{1}{2^{n}} P_{T}^{n}(x, \cdot), \quad \forall x \in \mathcal{L},
$$

where $\left(P_{T}^{n}(x, \cdot)\right)_{n \geq 1}$ is defined recursively as follows

$$
P_{T}(x, A):=P_{T} I_{A}(x), \quad P_{T}^{n}(x, A):=\int_{\mathcal{L}} P_{T}^{n-1}(x, \mathrm{~d} y) P_{T}(y, A), \quad n \geq 2 .
$$

Let ' $(\mu, v)$ denote the set of all couplings of $\mu$ and $v$, where $\mu$ and $v$ are two given probability on $\mathcal{L}$, and $W_{2}^{\delta}(\mu, v)$ be the $L^{2}$-Wasserstein distance between them, i.e.

$$
W_{2}^{\delta}(\mu, v)=\inf _{\pi \epsilon^{\prime}(\mu, v)} \int_{\mathcal{L}} \int_{\mathcal{L}}\|x-y\|_{\infty}^{2} \pi(\mathrm{d} x, \mathrm{~d} y)
$$

where $\delta(x, y)=\|x-y\|_{\infty}$ is a distance on $\mathcal{L}$.

Corollary 3.3. Assume that (H1) and (H2) hold and $\mu$ satisfies $(\mathrm{H} 4)(\widetilde{K}=1)$. Then the following entropy-cost inequality holds for each $T>r$ and $f \in \mathscr{B}_{b}^{+}(\mathcal{L})$ with $\mu(f)=1$,

$$
\mu\left(P_{T}^{*} f \log P_{T}^{*} f\right) \leq \rho^{\prime}(T, r, H) W_{2}^{\delta}(\mu, f \mu),
$$

where $P_{T}^{*}$ is the adjoint operator of $P_{T}$ in $L^{2}(\mu)$ and

$$
\rho^{\prime}(T, r, H)=\left\|\sigma^{-1}\right\|_{\infty}\left(K_{2}+K_{2} e^{K_{1} T}+\frac{K_{1}}{1-e^{-K_{1}(T-r)}}\right)^{2} \frac{T^{2-2 H}}{2-2 H} .
$$

Proof. By Corollary 3.2 for $P_{T}^{*} f$, we have

$$
\begin{aligned}
P_{T}\left(\log P_{T}^{*} f\right)(\xi) & \leq \log P_{T}\left(P_{T}^{*} f\right)(\eta)+\rho(T, r, H, \xi, \eta) \\
& \leq \log P_{T}\left(P_{T}^{*} f\right)(\eta)+\rho^{\prime}(T, r, H)\|\xi-\eta\|_{\infty}^{2}, \quad \forall \xi, \eta \in \mathcal{L} .
\end{aligned}
$$


Integrating both sides of (3.14) with respect to $\pi \in \varphi(\mu, f \mu)$, we get

$$
\mu\left(P_{T}^{*} f \log P_{T}^{*} f\right) \leq \mu\left(\log P_{T}\left(P_{T}^{*} f\right)\right)+\rho^{\prime}(T, r, H) \int_{\mathcal{L}} \int_{\mathcal{L}}\|\xi-\eta\|_{\infty}^{2} \pi(\mathrm{d} \xi, \mathrm{d} \eta) .
$$

Note that, the Jensen's inequality and the hypotheses imply

$$
\mu\left(\log P_{T}\left(P_{T}^{*} f\right)\right) \leq \log \mu\left(P_{T}\left(P_{T}^{*} f\right)\right) \leq \log \mu\left(P_{T}^{*} f\right)=\log \mu\left(f P_{T} 1\right)=\log \mu(f)=0 .
$$

So, we get

$$
\mu\left(P_{T}^{*} f \log P_{T}^{*} f\right) \leq \rho^{\prime}(T, r, H) \inf _{\pi \epsilon^{\prime}(\mu, f \mu)} \int_{\mathcal{L}} \int_{\mathcal{L}}\|\xi-\eta\|_{\infty}^{2} \pi(\mathrm{d} \xi, \mathrm{d} \eta) .
$$

The proof is complete.

\section{Acknowledgement}

This research is partially supported by the NNSF of China (No. 61273179) and Natural Science Foundation of Hubei Province (No. 2016CFB479).

\section{References}

[1] Wang F. Y., Logarithmic Sobolev inequalities on noncompact Riemannian manifolds. Probab. Theory Relat. Fields. 109 (1997), 417-424.

[2] Aida S., Kawabi H., Short time asymptotics of certain infinite dimensional diffusion process. Stochastics Analysis and Related Topics. 48 (2001), 77-124.

[3] Aida S., Zhang T., On the small time asymptotics of diffusion processes on path groups. Potential Anal. 16z (2002), 67-78.

[4] Wang F. Y., Harnack inequality and applications for stochastic generalized porous media equations. Ann. Probab. 35 (2007), 1333-1350.

[5] Röckner M., Wang F. Y., Harnack and functional inequalities for generalized Mehler semigroups. J. Funct. Anal. 203 (2003), 237-261.

[6] Abdelhadi E. S., Renesse M. Von, Scheutzow M., Harnack inequality for functional SDEs with bounded memory. Electronic Communications in Probability. 14 (2009), 560-565.

[7] Wang F. Y., Yuan C., Harnack inequality for functional SDEs with multiplicative noise and applications. Stochastic Processes and their Applications. 121 (2011), 2692-2710.

[8] Liu W., Harnack inequality and applications for stochastic evolution equations with monotone drifts. Journal of Evolution Equations. 9 (2009), 747-770.

[9] Ouyang S. X., Harnack inequalities and applications for stochastic equations. Ph. D. Thesisi, Bielefeld University. 2009.

[10] Röckner M., Wang F. Y., Supercontractivity and ultracontractivity for (non-symmetric) diffusion semigroups on manifolds. Forum Math. 15 (2003), 893-921.

[11] Wang F. Y., Harnack inequalities for log-Sobolev functions and estimates of log-Sobolev constants. Ann. Probab. 27 (1999), 653-663.

[12] Wang F. Y., Logarithmic Sobolev inequalities: conditions and counterexamples. J. Operator Theory. 46 (2001), 183-197.

[13] Bobkov S. G., Gentil I., Ledoux M., Hypercontractivity of Hamilton-Jacobi equations. J. Math. Pures Appl. 80 (2001), 669-696. 
[14] Gong F. Z., Wang F. Y., Heat kernel estimates with applications to compactness of man-ifolds. Quart. J. Math. 52 (2001), 171-180.

[15] Wang F. Y., Harnack inequalities on manifolds with boundary and applications. J. Math. Pures Appl. 94 (2010), 304-321.

[16] Kawabi H., Functional inequalities and an application for parabolic stochastic partial differential equations containing rotation. Bull. Sci. Math. 128 (2004), 687-725.

[17] Arnaudon M., Thalmaier A., Wang F. Y., Harnack inequality and heat kernel estimates on manifolds with curvature unbounded below. Bull. Sci. Math. 130 (2006), 223-233.

[18] Prato G. Da, Röckner M., Wang F. Y., Singular stochastic equations on Hilbert spaces: Harnack inequalities for their transition semigroups. J. Funct. Anal. 257 (2009), 992-1017.

[19] Liu W., Wang F. Y., Harnack inequality and strong Feller property for stochastic fast diffusion equations. J. Math. Anal. Appl. 342 (2008), 651-662.

[20] Mandelbrot BB., Ness J. Van, Fractional Brownian motion, fractional noises and applications. SIAMRev. 10 (4) (1968), 422-437.

[21] Comte F., Renault E., Long memory continuous time models. J. Econometrics. 73 (1) (1996), 101-149.

[22] La F. De, Perez-Samartin AL., Matnez L., Garcia MA., Vera-Lopez A., Long-range correlations in rabbit brain neural activity. Ann Biomed Eng February. 34 (2) (2006), 295-299.

[23] Willinger W., Leland W., Taqqu M. and Wilson D., On self-similar nature of ethernet traffic. IEEE/ACM Trans Networking. 2 (1) (1994), 1-15.

[24] Rypdal M., Rypdal K., Testing hypotheses about sun-climate complexity linking. Phys Rev Lett. 104 (12) (2010), 128-151.

[25] Simonsen I., Measuring anti-correlations in the nordic electricity spot market by wavelets. Physica A. 322 (1) (2003), 597-606.

[26] Fan X. L., Derivative formula, integration by parts formula and applications for SDEs driven by fractional Brownian motion. arXiv:1206.0961.2012.

[27] Fan X. L., Harnack type inequalities and applications for SDE driven by fractional Brownian motion. Stochastic Analysis and Applications. 32 (2014), 602-618.

[28] Fan X. L., Integration by parts formula and applications for SDE driven by Fractional Brownian motion. Stochastic Analysis and Applications. 33 (2015), 199-212.

[29] Thao T. H., An approximation approach fractional analysis for finance. Nonlinear Analysis. 7 (2006), 124-132.

[30] Coutin L., An introduction to stochastic calculus with respect to fractional Brownian motion, In: Séminaire de probabilités XL. Berlin Heidelberg: Springer-Verlag, 2007, 3-65.

[31] Dung N. T., Semimartingale approximation of fractional Brownian motion and its application. Comput. Math. Appl. 61 (7) (2011), 1844-1854.

[32] Dung N. T., On delayed logistic equation driven by fractional Brownian motion. J. Comput Nonlinear Dyn. 7 (2012), 031005.

[33] Nualart D., Ouknine Y., Regularization of differential equations by fractional noise. Stochastic Processes and their Applications 102 (2002), 103-116.

[34] Röckner M., F Wang. Y., Log-Harnack inequality for stochastic differential equations in Hilbert spaces and its consequences. Infin. Dimens. Anal. Quantum Probab. Relat. Top. 13 (2010), 27-37.

[35] Fan X. L., Harnack inequality and derivative formula for SDE driven by fractional Brownian motion. Sci. China Math. 56 (2013), 515-524. 